\title{
On the Computer Simulation of Microparticles Capture in Magnetic Filters using OpenMP
}

\author{
C. Chantrapornchai \\ Department of Computing \\ Faculty of Science, Silpakorn \\ University, Nakhon-Pathom, \\ Thailand
}

\author{
S. Dainpraprai \\ Department of Computing \\ Faculty of Science, Silpakorn \\ University, Nakhon-Pathom, \\ Thailand
}

\author{
O. Wongtaweesap \\ Department of Computing \\ Faculty of Science, Silpakorn \\ University, Nakhon-Pathom, \\ Thailand
}

\begin{abstract}
In this paper, we present the computer simulation of motions of micro-size solid wastes particles dispersed in water. The physical theory describing dynamics of particles is used. Particles equations of motion are formulated and are solved numerically by using the standard fourth-order Runge-Kutta method to obtain the micro-particle trajectories. Many particle trajectories are traced out to investigate capture behavior. Computing of each trajectory is optimized by using variable time step scheme. Overall simulation procedures are speedup by using parallel algorithm based on OpenMp. All trajectory computation is distributed to a group of computing threads. Each thread computes, in each time step, its occupied trajectories in parallel. After all trajectories are computed, a master thread is dedicated as the rendering thread for displaying all trajectories using OpenGL.
\end{abstract}

\section{Keywords}

High gradient magnetic separation, Trajectories simulation, OpenMp, OpenGL.

\section{INTRODUCTION}

The capture of micro-size particles by high gradient magnetic separation (HGMS) is applied in many works such as the treatment of waste water from the factory [1], the capture of specific cells from biological suspensions [2]. The capture behaviour depends on many parameters such as the magnetic property of the particle, the strength of the applied magnetic field, particle size, fluid flow velocity, the material and the size of the magnetic collectors contained within the filters. By using the simulation, users of HGMS technique can vary operating parameters easily and choose the appropriate set of operating parameters that optimize the capture process.

In this paper, we present the computer simulation of capturing micro-size particles of solid wastes dispersed in water using magnetic filters. A set of parameter values is chosen and then simulated to view the particle trajectories. We propose some optimization to the computation together with the use of the parallel computing to speed up the computation of the trajectories. The simulation results are integrated in the visualization tool to help users of HGMS technique investigate trajectories and capture behaviour. The system will be useful to the scientists in many areas the industries. The work also demonstrates the use of parallel computing in the computational physics.

In the real applications of HGMS, the filters consist of many layers of ferromagnetic wires. Each layer of the wires is able to be pulled out from the filter independently for cleaning when it is fully loaded by captured particles and then pushed back into the filter for continuous operation. Figure 1 illustrates the operation of magnetic filters. In figure 1, the rectangular represents the canister of the filter. In the rectangular, the dashed lines separate layers of ferromagnetic wires and the black dots represent the cross section of the wires. Fluid of dispersed particle is fed into the filter. The magnetic field $\stackrel{\mathrm{H}}{H}_{0}$ is applied perpendicular to wires axes to magnetize the wires and produce magnetic interaction between the wires and particle [3]. Some particles are captured in the filter and fluid with lower particle concentration exit the filter.

If the target particles to be capture are weakly magnetic or non-metallic particles of micro-size then magnetic and fluid drag forces overwhelm other forces and effects such as inertial force, particle diffusion etc. and capture behaviours are investigated by analyzing particles' trajectories. The so called trajectory-analysis method is used for estimating capture probability of each particle by a wire in the layer then the capture efficiency of each layer and filter efficiency can be predicted. Some examples of predicting filter efficiency via trajectory-analysis method can be seen in many works such as the analyitcal study of magnetic targeting of drug loaded particles in microvasculatures [4], the simulation of drug carriers captured by ferromagnetic stent implanted in the blood vessel $[5,6]$, the study of microparticle captured by random wires in magnetic filters [7].

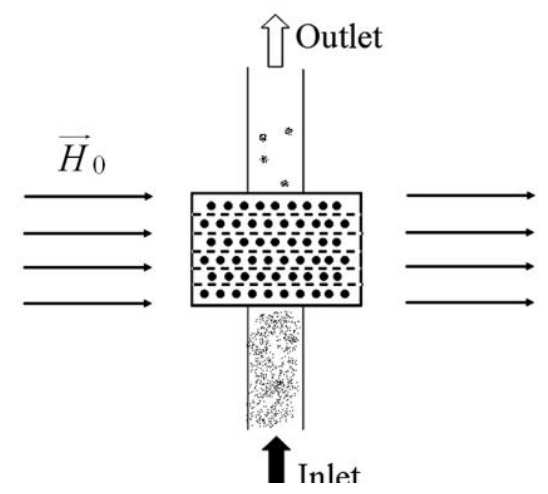

Fig 1: The operation of magnetic filters

To simulate accurate trajectories, the simulation time step used in numerical computing should be small enough. However, the small time step causes the long simulation time and large amount of particle coordinates data. In this work, the calculation of each trajectory can be optimized by using varying time step scheme and the overall simulation can be speedup by implementing parallel simulation based on OpenMp to obtain accurate results within the shortest time. Our method will help the users of HGMS technique 
conveniently adjust proper operating parameters by investigating the simulation and the visualization of the simulation results.

\section{THEORY OF MICROPARTICLES CAPTURE BY HGMS}

Figure 2 illustrates the mechanism of particles captured by a ferromagnetic wire in the filter. In Figure 2, a uniform external magnetic field is applied normally to the wire axis. The existence of the wire disturbs the uniformity of the background field and causes high gradient of the field close to wire surface. Particles in these regions experiences magnetic traction force which can be expressed as [8]

$$
\stackrel{\mathbf{u}}{F}_{m}=\mu_{0} \chi V_{p} H \stackrel{\mathbf{u}}{\nabla}(H),
$$

where $\mu_{0}, \chi=\chi_{p}-\chi_{f}, V_{p}$, and $H$ are the magnetic permeability of free space, the relative magnetic susceptibility between the particle and the fluid, the volume of a particle, the strength of local magnetic field at particle position, respectively. For weakly magnetic or non-metallic particles of micro-size, the value of $\chi_{p}-\chi_{f}$ is usually small $\left(\approx 10^{-3}-10^{-6}\right)$, volume of a particle is in the order of $10^{-8}$ $\mathrm{m}^{3}$ and the magnetic field strength $H$ cannot be increased above an order of $10^{6} \mathrm{~A} / \mathrm{m}$. Consequently, the magnitude of magnetic force $\stackrel{\mathrm{u}}{F}_{m}$ sufficient for capturing the particle requires a high value of $\stackrel{\mathrm{u}}{\nabla}(H)$, then $\stackrel{\mathrm{u}}{F}$ overwhelms over other forces and processes and particles are able to be captured on the wire.

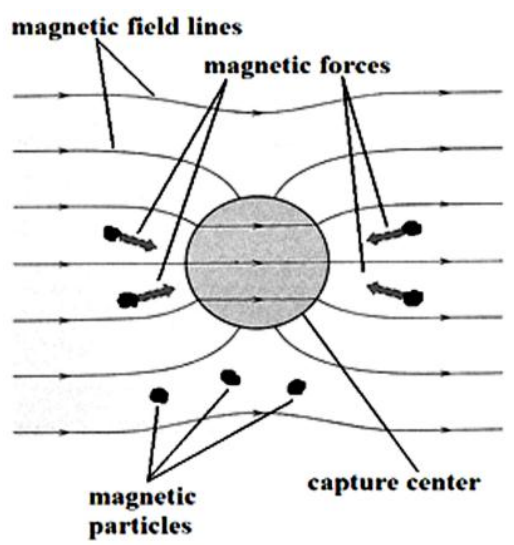

Fig 2: Mechanism of HGMS [9]

In this work, we consider the case of the low volume packing fraction of ferromagnetic wires contained within the magnetic filter (about 1\%). This means that wires in each layer shown in figure 1 are rather far from each others so each layer is not quickly saturated by trapped particles. Because of rather far distance between wires, it is considered that capture process of each wire is not affected by other wires and the single-wire approximation is considered.

Figure 3 illustrates the configuration of the problem in this work. In figure 3 , the origin of Cartesian coordinates is located at the center of a ferromagnetic wire. A uniform magnetic field $\stackrel{\mathrm{um}}{H}_{0}$ is applied in the positive $x$ direction. A potential flow of micro-size solid waste particle suspension is fed toward the wire with the entrance velocity $\stackrel{u}{V}_{0}$ in the direction that makes an angle $\alpha$ measured counter clock-wise to the positive $\mathrm{x}$ axis. For the case of $\alpha=0^{\circ}$, the incoming suspension flow parallel to $\stackrel{\mathrm{H}}{H}_{0}$ and the operation is called the longitudinal mode HGMS. For $\alpha=90^{\circ}$, the incoming suspension flow perpendicularly to $\stackrel{\mathrm{HI}}{H}_{0}$ and the operation is called the transverse mode HGMS. According to the geometry of the wire, we use coordinates $(r, \theta)$ for computing magnetic and suspension flow fields around the wire. The length of the wire is considered much larger than wire diameter so the magnetic and flow fields of suspension around each wire can be considered invariant along wire length. Consequently, we can treat the problem as two dimensional one and investigate the particle trajectories on a plane perpendicular to wire axis.

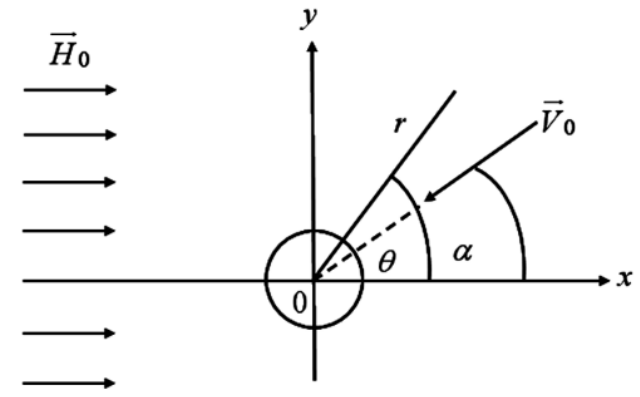

Fig 3: Configuration of the problem

Motion of a particle is governed by Newton; equation of motion that magnetic $\left(\stackrel{\mathrm{u}}{F_{m}}\right)$ and fluid drag forces $\left(\stackrel{\mathrm{u}}{F} v_{v}\right)$ are dominant forces.

$$
\stackrel{\mathrm{u}}{F}_{m}+\stackrel{\mathbf{u}}{F_{v}}=\stackrel{\stackrel{\mathbf{l}}{a}}{ },
$$

where $m$ and $a$ are particle mass and acceleration vector, respectively. After determining expression of the magnetic field around the wire [3] and use the expression in (1), the radial and angular components of magnetic force $\stackrel{\mathbf{u}}{F}_{m}$ can be expressed as [3],

$$
\begin{aligned}
F_{m r} & =-\frac{4 \pi \mu_{0} \chi M_{s} a^{2} b_{p}^{3}}{3 r^{3}}\left(\frac{M_{s} a^{2}}{2 r^{2}}+H_{0} \cos 2 \theta\right) \\
F_{m \theta} & =-\frac{4 \pi \mu_{0} \chi M_{s} a^{2} b_{p}^{3}}{3 r^{3}} H_{0} \sin 2 \theta,
\end{aligned}
$$

where $a, b_{p}$ and $M_{s}$ are wire radius, particle radius and magnetization of the wire, respectively. The fluid drag force $\stackrel{\mathrm{u}}{F_{v}}$ is assumed to satisfy Stokes' law,

$$
\begin{gathered}
F_{v r}=-6 \pi \eta b_{p}\left(\frac{d r}{d t}-V_{r}\right), \\
F_{v \theta}=-6 \pi \eta b_{p}\left(r \frac{d \theta}{d t}-V_{\theta}\right),
\end{gathered}
$$

where $\eta$ is suspension dynamic viscosity, $V_{r}$ and $V_{\theta}$ are radial and angular component of suspension velocity, respectively, and can be expressed as [3] 


$$
\begin{aligned}
& V_{r}=V_{0}\left(1-\frac{a^{2}}{r^{2}}\right) \cos (\theta-\alpha), \\
& V_{\theta}=-V_{0}\left(1+\frac{a^{2}}{r^{2}}\right) \sin (\theta-\alpha) .
\end{aligned}
$$

By substituting (3) - (5) in (2) and neglecting particle inertia, the particle equations of motion in radial and angular direction are [3]

$$
\begin{aligned}
\frac{d r_{a}}{d t} & =V_{0 a}\left(1-\frac{1}{r_{a}^{2}}\right) \cos (\theta-\alpha)-V_{m a}\left(\frac{K}{r_{a}^{5}}+\frac{\cos 2 \theta}{r_{a}^{3}}\right), \\
\frac{d \theta}{d t} & =-V_{0 a}\left(1+\frac{1}{r_{a}^{2}}\right) \frac{\sin (\theta-\alpha)}{r_{a}}-V_{m a} \frac{\sin 2 \theta}{r_{a}^{4}} .
\end{aligned}
$$

where

$$
V_{m a}=V_{m} / a=\frac{2 \chi \mu_{0} M_{s} H_{0} b_{p}^{2}}{9 \eta a^{2}},
$$

is called the normalized magnetic velocity, $r_{a}=r / a, K=$ $M_{s} / 2 H_{0}$ and $V_{0 a}=V_{0} / a$. Equations (6a) and (6b) obtained by using the magnetic field around the considered wire calculated based on the single-wire approximation. For the case of higher packing fraction, the magnetic effects from other wires are considered and the quantity $V_{m a}$ is easily modified by only one multiplying factor [7]. In the simulation, particle start positions are assigned and particle trajectories are obtained by solving Equations (6a)-(6b) numerically using the standard the fourth-order Runge-Kutta method and capture behaviours can be investigated.

\section{Optimization of single trajectory computing using varying time steps}

To solve Equations (6a) and (6b) accurately by using fourthorder Runge-Kutta method, the time step $\Delta t$ should small enough. Figure 4 shows the effect of using different time step sizes. In figure 4 , distances in $\mathrm{x}$ and $\mathrm{y}$ directions are measured in unit of wire radius and is denoted by a subscript $a$. Two particle trajectories, for transverse mode HGMS, starting from the same position at $\left(x_{a}=2.0, y_{a}=10.0\right)$, using different time step sizes are compared. It is observed that trajectories No. 1 which corresponding to time step sizes $\Delta t_{l}=1 \times 10^{-3} \mathrm{~s}$ is fail while No. 2 which corresponding to $\Delta t_{2}=1 \times 10^{-4} \mathrm{~s}$ is correct. So $\Delta t_{l}=1 \times 10^{-3} \mathrm{~s}$ is not sufficiently small to obtain the accurate trajectory. From the simulation experiments, the time step size should not larger than $\Delta t_{2}=1 \times 10^{-4} \mathrm{~s}$. This is because when a particle travels toward the wire (decreasing $r_{a}$ ) particle velocity $(V)$ increases rapidly as shown in figure 5 and high particle velocity means that the particle travels through long distance within a very small time interval. However, using too small time step size causes longer simulation time and larger amount of data to be manipulated. This may causes some problems when the number of computed trajectories becomes large and the computed trajectories are needed to be visualized.

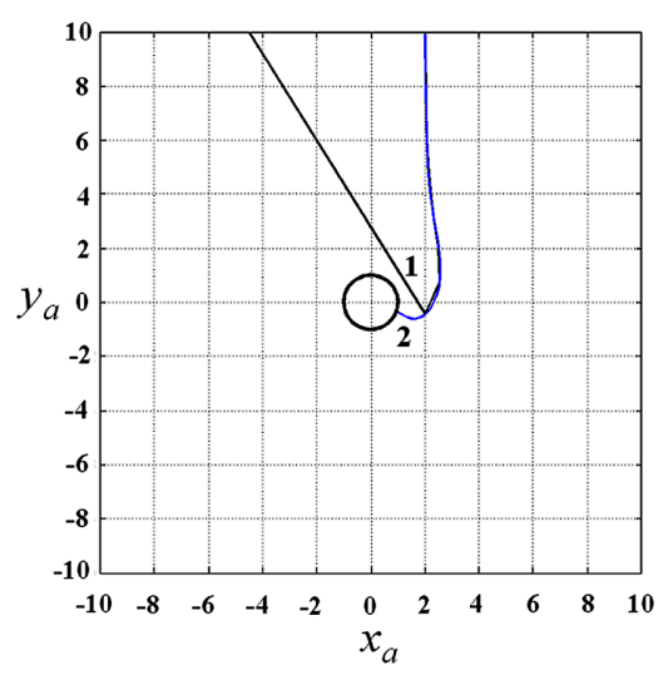

Fig. 4: The effect of different time steps.

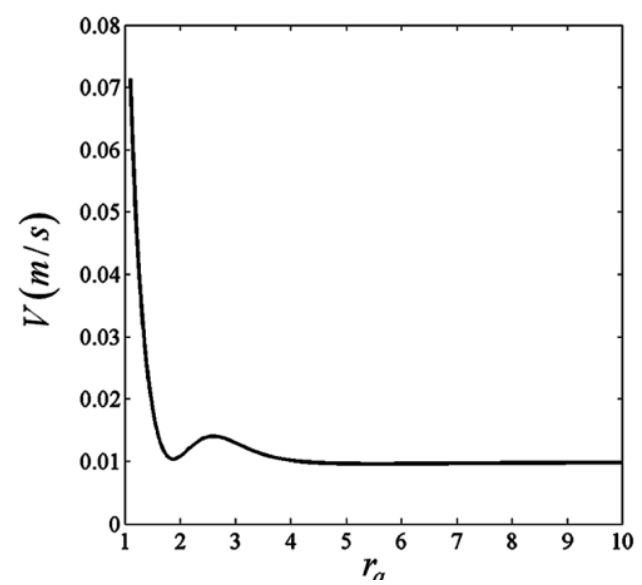

Fig. 5: The variation of particle velocity with the radial position start from position $\left(x_{a}=2.0, y_{a}=10.0\right)$.

To obtain accurate trajectories while the simulation time and manipulated data can be reduced. We introduce the use of varying time step scheme. We assign $\Delta s_{\min }$ as the limited distance that a particle can travels within a time step. The value of $\Delta s_{\min }$ should be some fractions, such as 0.1 or 0.5 of the particle diameter. At the start position, the particle initial velocity is set as fluid entrance velocity and instantaneous time step is

instantaneous time step $=\frac{\Delta \mathrm{s}_{\min }}{\text { instantaneous velocity }}$.

After first computational round, the new particle position is calculated. Then new particle instantaneous velocity and time step size are calculated by using Equation (8). This process continues until a trajectory is ended. By using this method accurate results are obtained while the simulation time and the amount of manipulated data can be reduced. 


\section{SIMULATION METHODOLOGY AND PARAMETERS}

The capture of micro-sized particles for both longitudinal and transverse modes HGMS are simulated. We formulate a square area domain surrounding a ferromagnetic wire. The domain is on a plane that perpendicular to wires axis. The width of the domain is 20 times of the wire radius. We set the normalized coordinates $\left(x_{a}, y_{a}\right)$ where $x_{a}=x / a$ and $y_{a}=y / a$ are distance in $x$ and $y$ direction measured in a unit of wire radius, respectively. Figures 6(a) and 6(b) illustrate the characteristics of longitudinal and transverse mode HGMS, respectively.

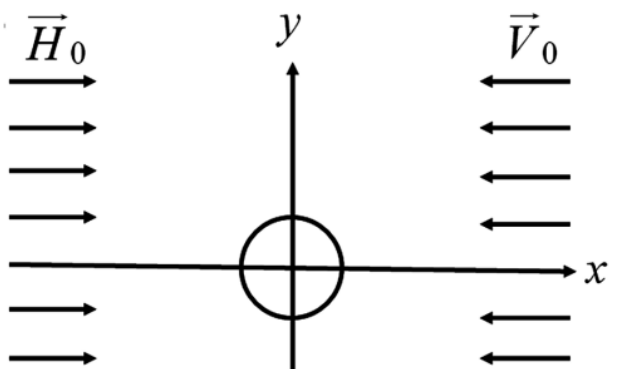

(a) The longitudinal mode HGMS

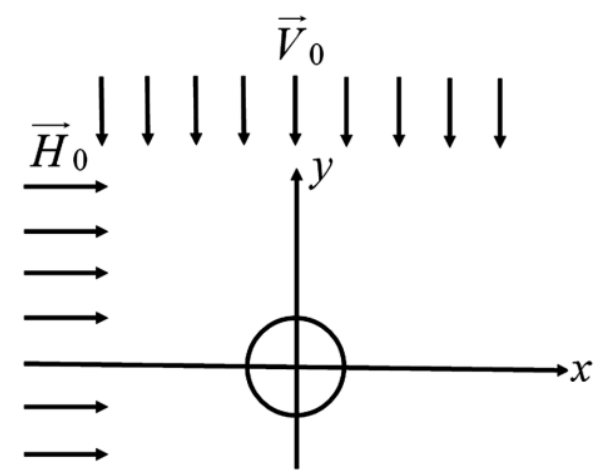

(b) The transverse mode HGMS

Fig 6: The characteristics of longitudinal and transverse modes HGMS

In the simulation, many particle starting positions $\left(x_{a 0}, y_{a 0}\right)$ in the Cartesian coordinates are assigned on the right and upper boundaries of simulation domain for longitudinal and transverse mode HGMS, respectively. Then the corresponding starting positions $\left(r_{a 0}, \theta_{0}\right)$ in cylindrical coordinates are determined by using the relations

and

$$
r_{a 0}=\sqrt{x_{a 0}^{2}+y_{a 0}^{2}}
$$

$$
\theta_{0}=\arccos \left(x_{a 0} / r_{a 0}\right) .
$$

Then particle positions in later times are computed by solving Equation (6a) and (6b) and particle trajectories can be traced out. The computing of each trajectory ends when the trajectory intercepts with wire boundary or one of simulation domain boundaries.

\subsection{Parameter setting}

The parameters of the simulation are operational parameter such as the strength of applied uniform magnetic field $H_{0}$, the entrance velocity $V_{0}$ of the suspension, material of the wire $\left(M_{s}\right)$, wire radius $(a)$. The example of solid waste particle in this work is Manganese Pyrophosphate $\left(\mathrm{Mn}_{2} \mathrm{P}_{2} \mathrm{O}_{7}\right)$ which is used in the production process of Lithium-Ion Batteries [10]. A suspension of $\mathrm{Mn}_{2} \mathrm{P}_{2} \mathrm{O}_{7}$ particles in water has the relative magnetic susceptibility $\chi=\chi_{p}-\chi_{f}=+2.03 \times 10^{-3}$ [11]. Particle radius is assigned as $1 \times 10^{-6} \mathrm{~m}$. The wire is stainless steel type so $M_{s}=1.397 \times 10^{6} \mathrm{~A} / \mathrm{m}$, of radius $a=10 \times 10^{-6} \mathrm{~m}$. The viscosity of the suspension is $1 \times 10^{-3} \mathrm{Ns} / \mathrm{m}^{2}$. The value $H_{0}$ is set as $8 \times 10^{5} \mathrm{~A} / \mathrm{m}$ (equivalent to the magnetic flux density $B_{0}=$ 1 Tesla), the entrance velocity $V_{0}$ is set as $0.01 \mathrm{~m} / \mathrm{s}$.

\subsection{Simulation domain decompositions}

In the parallel simulation, all start positions on the incoming boundary are divided and distributed to various computing threads by the master thread. The decompositions of starting positions are either row-wise or column-wise for longitudinal or transverse mode HGMS, respectively. Then each computing thread computes its occupied trajectories in parallel and independently. After all computing threads finish their computing works, data of particle coordinates in all trajectories are sent to the master thread which is dedicated as the rendering thread using OpenGL for the display. The configuration of computing works distribution is shown in Figure 7.

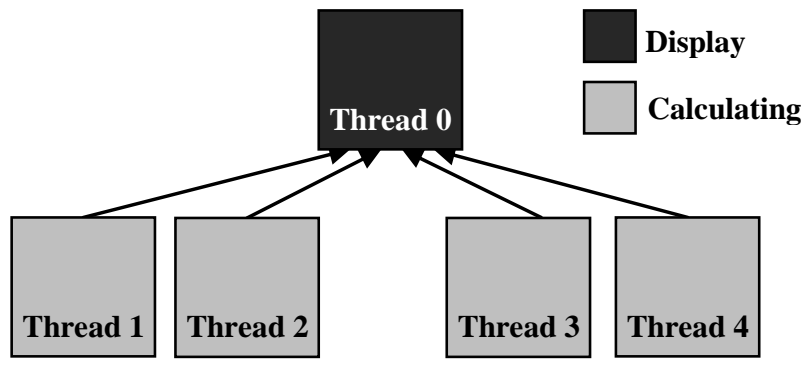

Fig 7: The configuration of work distribution by OpenMP

The simulation domain can be decomposed by using two approaches. In the first approach, the total simulation domain is decomposed into many consecutive areas. For this approach, total length of considered domain boundary is divided and assigned to each computing thread separately. Each computing thread computes its occupied trajectories from starting positions within its occupied domain length. In the second approach, simulation domain is decomposed in the configuration that computing threads occupy the interleaving areas. For the second approach, the first computing thread occupies the first start position then the next start positions are assigned to the next computing threads until the last computing thread is assigned. Then the assignment of start position progress in the periodic feature until the last start position is reached. Figures $8(a)$ and $8(b)$ illustrate the configurations of simulation domain decomposition by using consecutive and interleaving area for the case of longitudinal mode HGMS and four computing threads.

In our experiments of parallel simulation, we use these two configurations of simulation domain decomposition and compare the results such as the computed particle trajectories, total time used for simulation and display all trajectories. Then we can investigate which is the most appropriate approach for domain decomposition. This is by the 
observation that the computational work for each trajectory is not the same.

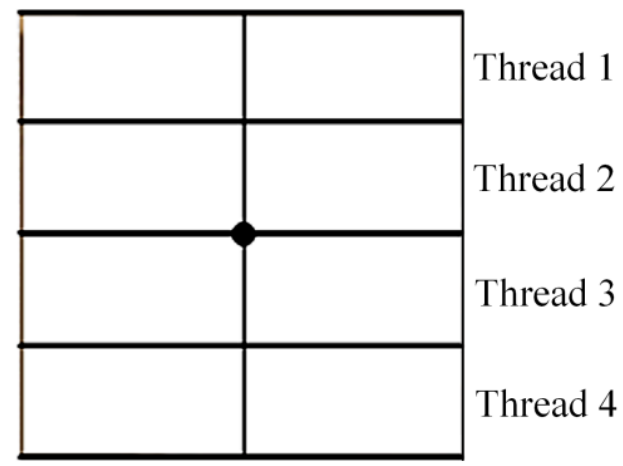

(a) Consecutive area decomposition

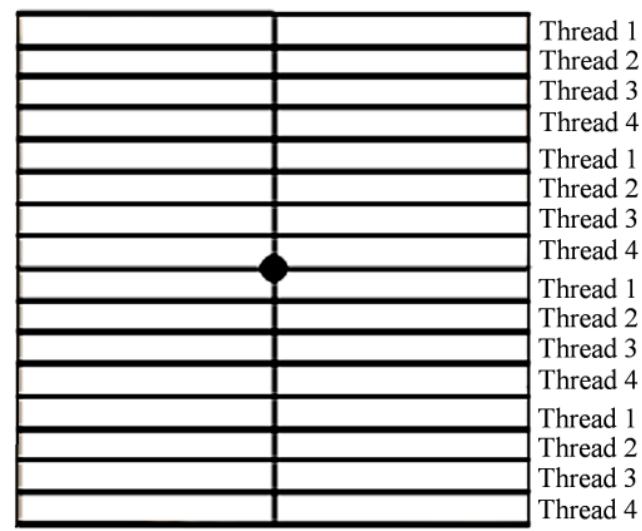

(b) Interleaving area decomposition

Fig 8: Two configurations of domain decomposition

\section{RESULTS AND DISCUSSION}

\subsection{Particle trajectories and capture \\ behaviors}

We compute and display many particle trajectory

within the simulation domain to investigate the capture behavior. We also investigate total time for trajectories computing and displaying to evaluate the efficacy of parallel algorithm based on OpenMP. Figures 9(a) and 9(b) show the computed particle trajectories for longitudinal and transverse mode HGMS, respectively. In Figure 9(a) it is seen that, for the particle capture using the longitudinal mode HGMS, certain amounts of particles traveling towards the wire are captured on the wire surface that is against the incoming particles. The residual particles which are not captured travel pass the wire and out of the simulation domain. For the transverse mode shown in Figure 9(b), certain amounts of the incoming particle are captured on two side surfaces of the wire and no capture occurs on the surface of the wire that is against the incoming particles.

To understand the capture behaviors observed in figure 9(a) and $(9 b)$, we consider the configuration of magnetic force that acts upon the particles in various regions around the wire shown in figure 10. In Figures 9(a), 9(b) and 10, it is seen that when the uniform magnetic field is applied in a positive $x$ direction, particles in regions on the top and the bottom of the wire experienced repulsive magnetic forces while particles in regions on the left and the right hand sides of the wire experienced attractive magnetic forces. Consequently, particles in regions on top and bottom of the wires are swept to the left side and the right hand side of the wire. Consider the particle capture in the longitudinal mode HGMS in Figure 9(a), the particles travel towards wire from the right hand side. Particles traveling in regions near to a positive $x$ axis experienced strong attractive magnetic and are brought to the right surface of the wire because the effect of the magnetic force overwhelms the effect of fluid convection. Incoming particles traveling in region far from the positive $x$ axis experiences the weak attractive magnetic force and are carried away from the wire since the effect of fluid convection is larger than the effect of magnetic force.

For particle capture in transverse mode HGMS in Figure 9(b), the incoming particles enter the upper boundary of the simulation domain. Incoming particles which are travel near to the positive $y$ axis experienced the strong magnetic force that sweeps the particles from the top side to be captured on the left side and the right side of the wire. Other incoming particles which travel far from the positive $y$ axis experienced weak sweeping magnetic force and are carried pass the wire by the fluid convection.

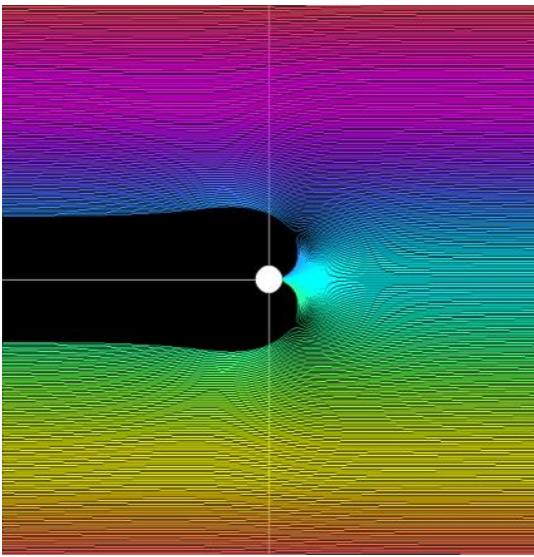

(a) The particle trajectories in the longitudinal mode HGMS

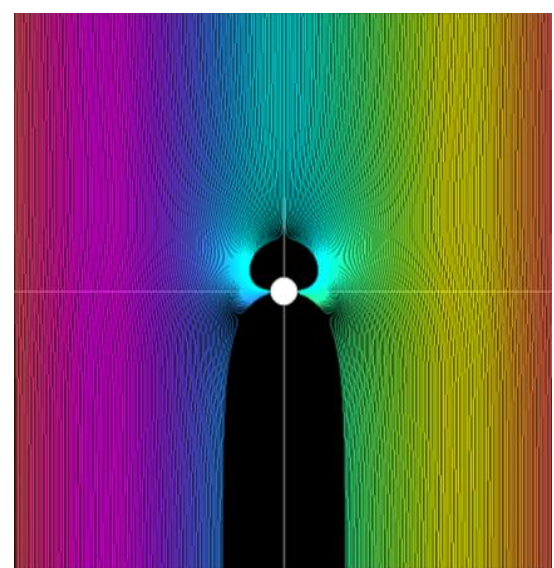

(b) The particle trajectories in the transverse mode HGMS

Fig 9: Particle trajectories in two modes of HGMS 


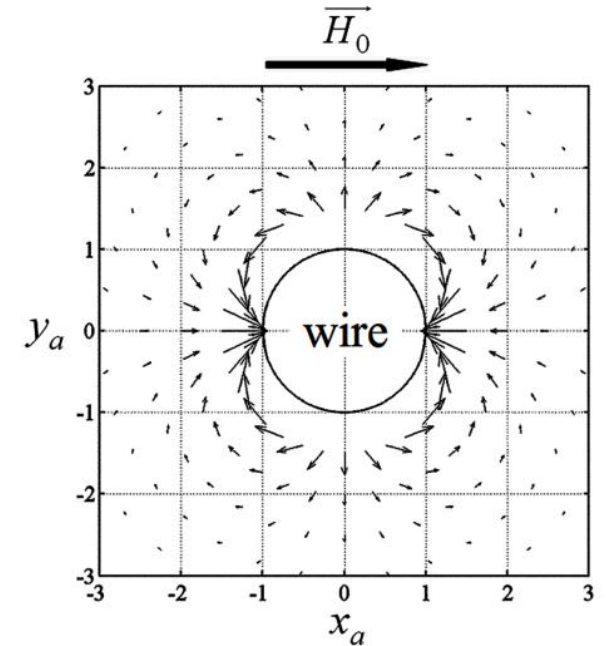

Fig 10: Direction magnetic force acting on particles in various regions around the wire [9]

From Figures 9(a) and 9(b), it is observed that particles are captured if and only if the start positions are in a certain range from flow symmetrical axis. We define the capture length, denoted by $R_{c}$, as the maximal distance from flow symmetrical axis that the particles entering the domain are captured on the wire. Figures 11(a) and 11(b) illustrate capture length for transverse and longitudinal modes HGMS respectively.

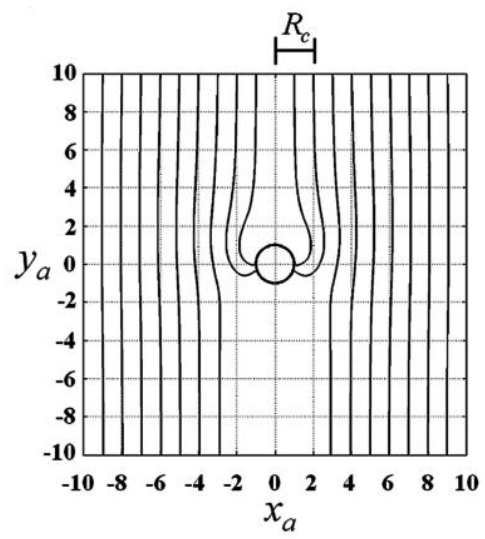

(a) Traverse mode HGMS

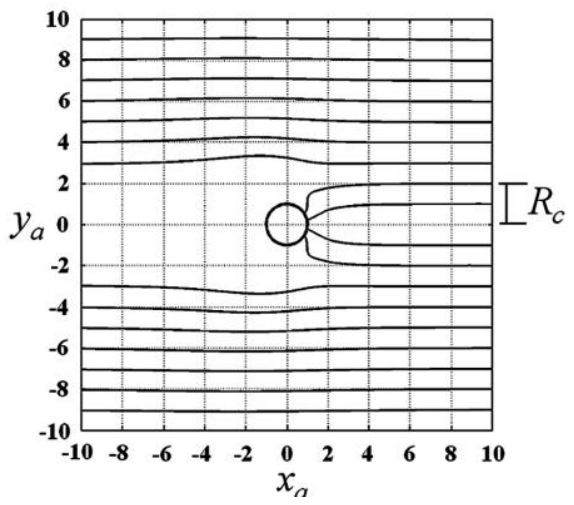

(b) Longitudinal mode HGMS

Fig 11: The capture lengths

\subsection{Efficiency of the optimization of single trajectory computing}

We investigate the efficacy of the optimization of single trajectory computing using varying time step scheme by comparing the number of simulation rounds and times measured from the method using fixed $\left(\Delta t=1 \times 10^{-6} \mathrm{~s}\right)$ and varying time step schemes. The improvement of computing performance can be observed. Tables 1 and 2 show the comparison of simulation rounds and times for the transverse and longitudinal modes for 9 start positions, respectively.

From Table 1, it is observed that the varying time step scheme decreases simulation time effectively for about one order of magnitude (in the $1^{\text {st }}$ to the $5^{\text {th }}$ rows of the table) when the start position is within a certain range from flow symmetrical axis. However, the simulations using fixed and varying time step schemes provide almost the same performance when the start position is beyond the certain range which equals to the capture length. Then the capture length is equal to two times of wire radius. Similarly, in Table 2 , the shown data have similar behaviour as that in Table 1 .

Table 1. Comparison of simulation rounds and times for transverse mode HGMS.

\begin{tabular}{|c|r|r|r|r|}
\hline \multirow{2}{*}{$\begin{array}{c}\text { Start } \\
\text { position }\end{array}$} & \multicolumn{2}{|c|}{ Simulation rounds } & \multicolumn{2}{c|}{ Simulation times $(\mathrm{s})$} \\
\cline { 2 - 5 } & \multicolumn{1}{|c|}{ fixed $\Delta t$} & varied $\Delta t$ & fixed $\Delta t$ & varied $\Delta t$ \\
\hline$(0.2,10)$ & $\mathbf{1 0 3 1 3}$ & $\mathbf{1 0 2 3}$ & $\mathbf{0 . 0 6 4}$ & $\mathbf{0 . 0 1 5}$ \\
\hline$(0.4,10)$ & $\mathbf{9 9 3 1}$ & $\mathbf{1 0 2 5}$ & $\mathbf{0 . 0 6 3}$ & $\mathbf{0 . 0 1 5}$ \\
\hline$(0.6,10)$ & $\mathbf{9 7 4 8}$ & $\mathbf{1 0 3 2}$ & $\mathbf{0 . 0 6 3}$ & $\mathbf{0 . 0 1 5}$ \\
\hline$(0.8,10)$ & $\mathbf{9 6 6 4}$ & $\mathbf{1 0 4 1}$ & $\mathbf{0 . 0 6 3}$ & $\mathbf{0 . 0 1 5}$ \\
\hline$(1,10)$ & $\mathbf{9 6 4 6}$ & $\mathbf{1 0 5 3}$ & $\mathbf{0 . 0 6 3}$ & $\mathbf{0 . 0 1 5}$ \\
\hline$(2,10)$ & $\mathbf{1 0 7 0 0}$ & $\mathbf{1 1 6 4}$ & $\mathbf{0 . 0 6 4}$ & $\mathbf{0 . 0 1 5}$ \\
\hline$(3,10)$ & 2001 & 2006 & 0.016 & 0.016 \\
\hline$(4,10)$ & 1989 & 2002 & 0.016 & 0.016 \\
\hline$(5,10)$ & 1987 & 2001 & 0.016 & 0.016 \\
\hline
\end{tabular}

Table 2. Comparison of simulation rounds and times for longitudinal mode HGMF.

\begin{tabular}{|c|r|r|r|r|}
\hline \multirow{2}{*}{$\begin{array}{c}\text { Start } \\
\text { position }\end{array}$} & \multicolumn{2}{|c|}{ Simulation rounds } & \multicolumn{2}{c|}{ Simulation times $(\mathrm{s})$} \\
\cline { 2 - 5 }$(10,0.2)$ & fixed $\Delta t$ & varied $\Delta t$ & fixed $\Delta t$ & varied $\Delta t$ \\
\hline$(10,0.4)$ & $\mathbf{7 9 8 5}$ & $\mathbf{8 8 8}$ & $\mathbf{0 . 0 4 7}$ & $\mathbf{0 . 0 1 5}$ \\
\hline$(10,0.6)$ & $\mathbf{8 0 7 9}$ & $\mathbf{8 9 0}$ & $\mathbf{0 . 0 4 7}$ & $\mathbf{0 . 0 1 5}$ \\
\hline$(10,0.8)$ & $\mathbf{8 1 6 4}$ & $\mathbf{8 9 8}$ & $\mathbf{0 . 0 4 7}$ & $\mathbf{0 . 0 1 5}$ \\
\hline$(10,1)$ & $\mathbf{8 2 8 1}$ & $\mathbf{9 0 4}$ & $\mathbf{0 . 0 4 7}$ & $\mathbf{0 . 0 1 5}$ \\
\hline$(10,2)$ & $\mathbf{1 0 6 1 4}$ & $\mathbf{9 8 5}$ & $\mathbf{0 . 0 6 3}$ & $\mathbf{0 . 0 1 5}$ \\
\hline$(10,3)$ & 1998 & 2004 & 0.016 & 0.016 \\
\hline$(10,4)$ & 1988 & 2002 & 0.016 & 0.016 \\
\hline$(10,5)$ & 1987 & 2001 & 0.016 & 0.016 \\
\hline
\end{tabular}

Figure 12 shows the comparison between varying and fixed $\left(\Delta t=1 \times 10^{-6} \mathrm{~s}\right)$ time steps size for computing a trajectory starting within the capture length. The comparison shows that the time step size can be varied from the order of $10^{-5} \mathrm{~s}$ down to $10^{-6} \mathrm{~s}$. Thus, with our optimization approach, the time step can be varied and the simulation time can be reduced.

Then we investigate the accuracy of trajectory computing using varying time step size. It is exactly true that using the very small time step size provides the accurate particle trajectories. When using the varying time step size from larger $\left(10^{-5} \mathrm{~s}\right.$.) down to smaller $\left(10^{-6} \mathrm{~s}\right.$.) values, we consider Equations (6a) and (6b). It is observed that the second group on the right hand side of Equations (6a) and (6b), which is 
caused by magnetic force, decreases with increasing $r_{a}$ very faster than the first group which is caused by suspension flow. Consequently, the first group dominates the computing when particle is far from the wire while the second group dominates only when particle is close to the wire. If a particles starts at a position outside the capture length, the effect from suspension flow always dominates the effect from magnetic force and particle velocity is very close to the suspension entrance velocity $V_{0}$ for every time step. The sufficiently small instantaneous time step can be determined by dividing the characteristic length (fraction of particle diameter) with characteristic velocity (suspension entrance velocity) which is about $2 \times 10^{-4} \mathrm{~s}$ for parameters stated in section 4.1. Consequently, the 2000 rounds computation (as seen in $7^{\text {th }}$ to $9^{\text {th }}$ row of Table I and II) using the time step $\Delta t=1 \times 10^{-5} \mathrm{~s}$ provides accurate results in the region beyond the capture length. If a particle starts within the capture length (as seen in $1^{\text {st }}$ to $6^{\text {th }}$ row of Table I and II), the effects from the suspension flow and magnetic force become comparable. Particle velocity increases continuously as shown in figure 5 . In each round of computing, the sufficiently small time step is computed by using the particle instantaneous velocity obtained from the contributions of both effects from suspension flow and magnetic force. Because the simulation round is significantly reduced, the accumulated errors are also be reduced. The stable and accurate trajectoreis can be obtained.

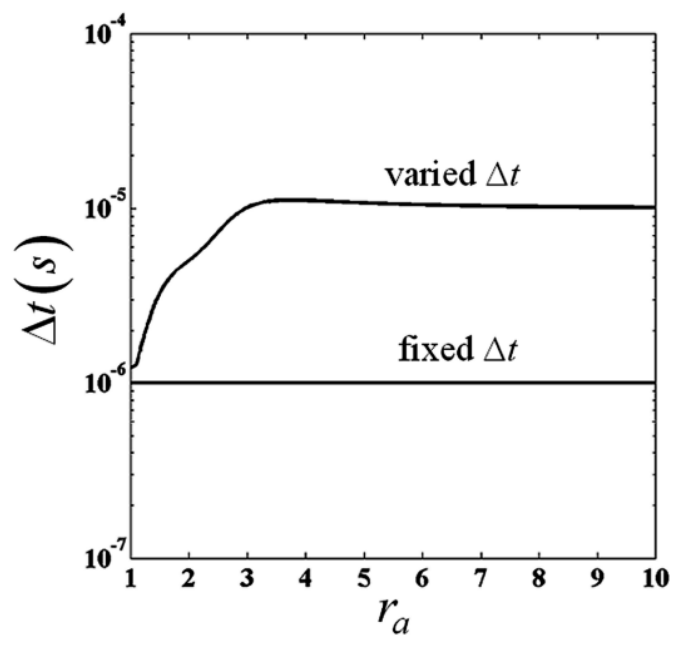

Fig 12: Comparisons of varied and fixed time steps.

In this paper, we develop a prototype of parallel simulation framework for exploring particle trajectories. The simulation is done by using OpenMP and MPI. We simulate in two modes: longitudinal mode and traverse mode. The equation solver of particle trajectory at a time step is solved using $4^{\text {th }}$ order Runge-Kutta method. The simulation results match with the literature for different magnetic fields and initial velocities of the particles cases. The simulation event is a 2-dimensional domain. We integrate the simulation into an interactive trajectory visualization framework by OpenGL. The tool is flexible in such a way that the initial parameters can be varied. Some parameters can be changed during the simulation. The simulation can be viewed at each time step. Partial trajectories can be viewed and the capturing results can be zoomed.

In the future, we will simulation in a 3-dimensional domain of event and uses GPU programming for increase efficency of tool. We will render paticles trajectory animation in a smooth frame rate while calculating the trajectories in real time.

From the experimental results, using the varying time provides stable and accurate simulation results of particle trajectories for main parameters $\left(H_{0}, M_{s}, V_{0}\right)$. If the trajectories are calculated in the region within the capture length, the simulation round can be decreased about 10 times, the simulation time and manipulated data can be decreased for about 3-4 times as seen in Tables 1 and 2. In the region beyond the capture length, the varying time step method has the same performance as the fixed time step method. Thus, the varying time step method gives benefits in overall for all regions in the computational domain.

\subsection{Time Efficiency of parallel simulation based on OpenMP}

The experiments show the performance of the parallel implementation using OpenMP. The computational domain is assigned to the threads in two styles: consecutive areas or interleaving areas. In the transverse mode, the area is divided in the column-wise style and in the longtitudinal mode, the area is divided in the row-wise style.

We assume the parameters in simulation as following: the amount of particles $=400$ particles (which means 400 start positions are assigned on incoming boundary of simulation domain), the initial velocity of particles $=0.01 \mathrm{~m} / \mathrm{s}$. We use the computer with 4 cores, $2.8 \mathrm{GHz}, 4 \mathrm{MB}$-cache and 8GB RAM for the simulation.

Table 4 shows the total time for simulation and displaying trajectories compared between two approaches of domain decomposition

Table 4. Total simulation time for consecutive and interleaving area division.

\begin{tabular}{|c|c|c|}
\hline Number of threads & $\begin{array}{c}\text { Time (s) } \\
\text { (Consecutive area) }\end{array}$ & $\begin{array}{c}\text { Time (s) } \\
\text { (Interleaving area) }\end{array}$ \\
\hline 2 & 178 & 174 \\
\hline 4 & 102 & 95 \\
\hline 8 & 83 & $\mathbf{7 7}$ \\
\hline 16 & 126 & 118 \\
\hline
\end{tabular}

We partition the work into 248 and 16 threads to calculate the trajectory. The best timing results use 8 threads. Comparing two subdomain decomposition, using the interleaving technique is more efficient than the consecutive way. This is because the particle trajectories are symmetrics in this testing case. Thus the interleaving manner gives the more balanced load. In the overall tests, the interleaving approach reduces the computation time at most $7 \%$. This is for the sample only 400 trajectories. The advantages will be more when more number of trajectories are explored.

\section{CONCLUSIONS}

We develop a prototype of the computer simulation for exploring micro-particle trajectories. The simulation is useful in the study of microparticle capture by bundles of ferromagnetic wires in magnetic filters. Due to the intensive computational work, the simulation is done using OpenMP. We simulate in two modes: longitudinal mode and traverse mode. The equation solver of particle trajectory are solved using $4^{\text {th }}$ order Runge-Kutta at the proposed varying time step 
fashion. The simulation results match with the literature for different magnetic fields and initial velocities of the particles cases. With the varying time step computation and parallel computation, the computation time can be reduced. The simulation event presentation is currently in a 2-dimensional domain.

We will integrate the simulation into an interactive trajectory visualization framework by OpenGL. The tool will be flexible in such a way that the initial parameters can be varied. Some parameters can be changed during the simulation. The simulation can be viewed at each time step. Partial trajectories can be viewed and the capturing results can be zoomed.

Also, in the future, we will simulate another mode of HGMS called the axial mode [3] which entering suspension flow parallel to wires axes. In this mode, simulation of particle trajectories is considered in three dimensions. Different from the transverse and longitudinal modes HGMS that only one value of capture length is determined, capture area composes of many values of capture length are determined in the axial mode and parallel computing become more necessary. Because of the developed technology of parallel computing using GPU, we consider in a three dimensional domain of event and uses GPU programming for increase efficency of tool [12-14]. We will render paticles trajectory animation in a smooth frame rate while calculating the trajectories in real time.

\section{ACKNOWLEDGEMENTS}

This work was supported in part by Silpakorn University Research and Development Institute and Faculty of Science Research Funding, Silpakorn University, Thailand

\section{REFERENCES}

[1] Nishijima, S., Takeda, S. I. Superconductin High Gradient Magnetic Separation for Purification of Wastewater From Paper Factory. IEEE Trans. Appl. Supercon. 16 (June 2006), 1142-1145.

[2] Inglis, D., Riehn, R., Sturm, J., Austin, R. H. Microfluidic High Gradient Magnetic Cell Separation. J. Appl. Phys. 99 (April 2006), 08K101-1-08K101-3.

[3] Gerber, R., and Birss R. R. 1983 High Gradient Magnetic Separation. John Wiley \& Sons.

[4] Furlani, E. P., Ng, K. C. Analytical model of magnetic nanoparticle transport and capture in the microvasculature. Phys. Rev. E 73 (June 2006), 061919$1-9$.

[5] Chen, H., Ebner, A. D., Rosengart, A. J., Kaminski, M. D., Ritter, J. A. Aanlysis of magnetic drug carrier particle capture by a magnetizable intravascular stent: 1 Parametric study with single wire correlation. J. Magn. Magn. Mater. 284 (December 2004), 181-194.

[6] Chen, H., Ebner, A. D., Kaminski, M. D., Rosengart, A. J., Ritter, J. A. Aanlysis of magnetic drug carrier particle capture by a magnetizable intravascular stent-2: Parametric study with multi-wire two-dimensional model. J. Magn. Magn. Mater. 293 (May 2005), 616632 .

[7] Natenapit, M., Sanglek, W. Capture radius of magnetic particles in random cylindrical matrices in high gradient magnetic separation. J. Appl. Phys. 85 (1999), 660-664

[8] Fletcher, D. Fine particle high gradient magnetic entrapment. IEEE Trans. Magn. 27 (July 1991),36553677.

[9] Hournkumnuard K., Chantrapornchai, C. Parallel simulation of concentration dynamics of nano-particles in high gradient magnetic separation. Simul. Model. Pract. Th. 19 (February 2011), 847-871.

[10] Zhou, H., Upreti, S., Chernova, N. A., Hautier, G., Ceder, G., Whittingham, M. S. Iron and Manganese Pyrophosphates as Cathodes for Lithium-Ion Batteries. Chem. Mater. 2 (December 2011), 293-300.

[11] Davies, L. P., Gerber, R. 2-D simulation of ultra-fine particle capture by a single-wire magnetic collector.

IEEE Trans. Magn. 26 (September 1990), 1867-1869.

[12] Paulius, M. 2009. 3D finite difference computation on GPUs using CUDA. In Proceeding of 2nd Workshop on

General Purpose Processing on Graphics Processing Units.

[13] Li, K., Kong, F. 2009. Parallel 3D finite difference time domain simulation on graphics processors with CUDA.

In Proceeding of International Conference on Computer Intelligence and Software Engineering.

[14] Khajeh-Saeed, A. Computational fluid dynamics simulations using many graphics processors. Comput. Sci. Eng. 14(3) (2012), 10-19. 